\title{
Traduction et réécriture chez Vladimir Nabokov : genèse d'une œuvre en trois langues
}

\section{Olga Anokhina}

\section{(2) OpenEdition}

1 Journals

\section{Édition électronique}

URL : http://journals.openedition.org/genesis/1306

DOI : 10.4000/genesis.1306

ISSN : 2268-1590

\section{Éditeur :}

Presses universitaires de Paris Sorbonne (PUPS), Société internationale de génétique artistique littéraire et scientifique (SIGALES)

\section{Édition imprimée}

Date de publication : 15 avril 2014

Pagination : 111-127

ISBN : 9782840509370

ISSN : 1167-5101

\section{Référence électronique}

Olga Anokhina, «Traduction et réécriture chez Vladimir Nabokov : genèse d'une œuvre en trois langues », Genesis [En ligne], 38 | 2014, mis en ligne le 29 juin 2016, consulté le 12 juin 2020. URL http://journals.openedition.org/genesis/1306 ; DOI : https://doi.org/10.4000/genesis.1306 


\title{
Traduction et réécriture chez Vladimir Nabokov : genèse d'une œuvre en trois langues*
}

Olga Anokhina

\begin{abstract}
Je suis un Russe tricolore, un Américain qui fut élevé en Angleterre, un Saint-Pétersbourgeois qui a un grasseyement parisien en russe, mais n' en a pas en français, où je roule plutôt mes $r$ à la façon russe.

Vladimir NABOKOV ${ }^{1}$
\end{abstract}

\section{La traduction comme prémices de l'écriture créative}

Dans notre contribution, nous allons nous intéresser à la pratique de traduction de Vladimir Nabokov (18991977). Comme on le sait, Nabokov écrivait, traduisait et s'autotraduisait en trois langues : en russe, en anglais et en français.

Élevé dans un cadre très privilégié par des parents anglophiles et des précepteurs de langues anglaise et française, Nabokov était trilingue dès son plus jeune âge. Il faut toutefois souligner le rôle prépondérant de l'anglais car c'est dans cette langue que Nabokov fait ses premières lectures. C'est donc en anglais qu'il entre dans le monde enchanté de la littérature.

En 1919, à l'âge de vingt ans, Nabokov quitte la Russie et débute ses études universitaires à Cambridge en anglais. L'anglais joue donc à nouveau un rôle important, celui des études supérieures. À Cambridge, il s'essaie à ses premières traductions vers la langue russe. L'une des premières traductions de Nabokov fut l'intraduisible Alice au pays des merveilles de Lewis Carroll, qu'il traduit de l'anglais vers le russe. Dans les années vingt, il a également traduit du français vers le russe des poèmes d'Alfred de Musset et de Rimbaud, ou encore Colas Brugnon de Romain Rolland.
Ces traductions lui permettent de s'exercer dans sa langue maternelle, de se confronter à sa matière linguistique, de chercher son style et vont de toute évidence préparer le terrain pour son écriture russe à venir ${ }^{2}$. Qu'il traduise du français ou de l'anglais, il traduit vers le russe qui sera sa langue d'expression dans les vingt prochaines années. Sur le plan cognitif, la traduction joue donc un rôle fort important puisqu'elle permet à l'écrivain d'entrer dans l'expression écrite «par procuration » - en tant que traducteur - avant de s'assumer pleinement en tant qu'écrivain. On l'observe pour Nabokov, mais c'est vrai pour bien d'autres écrivains plurilingues ${ }^{3}$.

\footnotetext{
* Nous remercions très chaleureusement Daniel Ferrer pour les échanges qui ont accompagné la rédaction de cet article.

1. V. Nabokov, cité dans P. Dommergues, « Nabokov n'appartient à personne », Magazine littéraire, n $^{\circ}$ 12, novembre 1967.

2. Nous l'avons expliqué en détail dans O. Anokhina, « Le rôle du multilinguisme dans l'activité créative de Vladimir Nabokov», dans O. Anokhina, Multilinguisme et créativité littéraire, Louvain-la-Neuve, Academia Bruylant/Harmattan, 2012, p. 15-25.

3. C'est par exemple le cas de Cioran ou de Wang Wen-hsing. Voir N. Cavaillès, "L'écriture et l'intraduisible. Le multilinguisme dans la genèse du Précis de décomposition de Cioran ", dans O. Anokhina, op. cit., p. 145-156; S. Marchand, «D'Hemingway au chinois classique : le travail de la langue de l'écrivain taïwanais Wang Wen-hsing », dans O. Anokhina, F. Davaille, H. Sanson (dir.), Continents manuscrits [en ligne], $\mathrm{n}^{\circ}$ 2, «Bien écrire/mal écrire : écrire en "périphérie" de la norme (écrivains plurilingues, écrivains "francophones" ", à paraître.
} 
Quatre ans plus tard, en 1923, il s'installe à Berlin, ville devenue terre d'accueil pour de nombreux artistes russes après la révolution de 1917. Immergé entièrement dans le milieu russophone, Nabokov commence à écrire dans sa langue maternelle : «L'histoire de ces années en Angleterre est en réalité l'histoire de mes efforts pour devenir un écrivain russe ${ }^{4}$. »

En l'espace de quatre ans, Nabokov publie trois romans en langue russe qui le consacrent comme l'un des écrivains russes les plus importants du Xxe siècle : Machenka (1926), Roi, dame, valet (1928), La Défense Loujine (1930).

Parallèlement à l'usage de la langue russe pour l'écriture et dans sa vie quotidienne, Nabokov utilise le français et l'anglais dans sa correspondance et lors des conférences à Paris et en Angleterre.

Pour désigner une langue « maternelle », on utilise en langue russe l'adjectif dérivé de «patrie » : langue paternelle, langue natale [родной язык]. Comme bien des écrivains émigrés du $\mathrm{xx}^{\mathrm{e}}$ siècle, Nabokov était dans une situation linguistique et affective très particulière, car son pays d'origine était pour lui inaccessible à jamais. Résidant sur le sol allemand, c'est la langue russe qui devient son refuge. Loin de sa terre natale, Nabokov assume pleinement son statut d'écrivain russe dont la production littéraire, qu'il s'agisse des œuvres originales ou des traductions, se fait dans sa langue maternelle.

À la fin des années trente, Nabokov quitte Berlin pour Paris. C'est alors une logique inverse qui s'installe dans son activité de traduction : Nabokov commence à traduire les auteurs russes vers l'anglais. Par ailleurs, il écrit directement en français 5 une nouvelle, Mademoiselle $O$, publiée à Paris en 19366. Malgré sa parfaite maîtrise du français, l'écriture dans cette langue ne sera qu'une courte parenthèse dans son activité littéraire car, en 1940, Nabokov quitte la France pour les États-Unis.

\section{Écrire et traduire en langue anglaise : nouveau refuge}

À son arrivée en Amérique, Nabokov écrit en anglais. Malgré son usage intensif du français les années précédentes, il a déjà commencé à préparer le terrain pour sa reconversion littéraire anglophone, puisque, dès les années trente, quelques-unes de ses œuvres russes ont été traduites et publiées en anglais. C'était une première période de traduction des œuvres de Nabokov vers l'anglais. Si quelques traductions ont été faites sans intervention de l'auteur ${ }^{7}$, on note déjà l'importance que Nabokov accorde à la possibilité d'intervenir sur la traduction de ses œuvres. Pour convaincre ses traducteurs de le laisser participer au processus créatif, Nabokov n'hésite pas à utiliser toutes sortes d'arguments :

\section{Lettre de 1937 à Gleb Struve}

Oui, il serait superbe que vous vous occupiez de la traduction de Pilgram! [Aurelian en trad. angl.] (mais avec ma participation active car cette nouvelle contient de l'entomologie qui peut vous donner du fil à retordre $)^{8}$.

Carte postale du 3 mai 1937 à Gleb Struve

Je n'ai pas le temps de traduire Printemps [à Fialta], mais si vous le traduisiez keeping in touch with, cela serait formidable. Denis Roche vient de finir la traduction française d'une de mes nouvelles : nous avons passé avec lui six heures en vérifiant chaque phrase ${ }^{9}$

4. «The story of my college years in England is really the story of my trying to become a Russian writer » (V. Nabokov, Speak, Memory, New York, G. P. Putnam's Sons, 1966, p. 261).

5. Sur la relation de Nabokov avec la langue française, voir O. Anokhina, "Nabokov et la langue française : étude de l'utilisation du français dans les œuvres publiées, les manuscrits, les traductions et la correspondance de Vladimir Nabokov », dans Vladimir Nabokov et la France, Actes du colloque international de l'université Paris IV-Sorbonne, en partenariat avec l'ENS, les 30, 31 mai et 1er juin 2013 (à paraître).

6. Nabokov a également écrit et publié en français un article sur Pouchkine, «Le vrai et le vraisemblable » (1937) dans La Nouvelle Revue française, et un article, «Les écrivains et l'époque » (1931), dans la revue Le Mois, reproduit dans M. Couturier, Nabokov ou la Tentation française, Paris, Gallimard, 2011.

7. Il s'agit de la traduction par Gleb Struve de deux nouvelles, The Return of Tchorb et The Passenger, parues en 1932 à Paris, ainsi que la nouvelle The Potato-Elf, traduite par Serge Bertenson et Irène Kosinska et publiée dans Esquire en 1939

8. « Да, было бы чудно, если бы вы перевели пильграма! (но с ближайшим моим участием, так как там есть энтомология с которой можно запутаться) » (Congress Library, Manuscript Division, Box 22, folder 6). Ici et plus loin notre traduction en français. 9. «У меня нету времени переводить “Весну”, но если бы вы перевели keеріng in touch with то было бы чудесно. Только что Denis Roche закончил франиузский перевод одной вещи и мы часов шесть с ним проверяли каждую фразу » (Congress Library, Manuscript Division, Box 22, folder 6). 
Dans ces deux courriers, Nabokov insiste sur le fait que le traducteur devra accepter sa «participation active » et traduire «keeping in touch with ». Il va jusqu'à évoquer - à titre d'exemple - son travail avec un autre traducteur en précisant qu'il a passé plusieurs heures à vérifier scrupuleusement chaque virgule dans une œuvre traduite.

Nous verrons plus loin que cette tendance à participer activement au processus de la traduction, déjà présente durant la période européenne de Nabokov, se renforcera à partir du moment où sa notoriété d'écrivain américain sera bien établie.

Une fois installé aux États-Unis, Nabokov acquiert la renommée d'un grand écrivain américain en l'espace de quelques années, en publiant notamment The Real Life of Sebastian Knight, Bend Sinister, Pnin, Lolita, Conclusive Evidence. Il doit alors faire face à un véritable engouement pour ses écrits et décide de traduire en anglais l'ensemble de son œuvre russe. Commence alors la deuxième période de traduction vers l'anglais qui deviendra l'une de ses préoccupations majeures de 1959 à 1972. Durant ces treize années, Nabokov collabore avec de nombreux traducteurs, comme Peter Pertzoff, Simon Karlinski, Michael Scammell, Michael Glenny et son fils Dimitri Nabokov.

Les traducteurs de Nabokov et l'écrivain lui-même ont souvent gardé leurs documents de travail, de sorte que nous pouvons observer aujourd'hui comment se déroulait le processus de traduction et de supervision par l'auteur. Bien que ces traductions aient été effectuées par des traducteurs de langue maternelle anglaise, elles ont toutes été minutieusement révisées par Nabokov lui-même. De ce fait, la frontière entre la traduction des œuvres de Nabokov et l'autotraduction est menue, tant la part de réécriture par l'auteur est importante sur le plan qualitatif et quantitatif 10 .

Nabokov a créé l'image d'un traducteur idéal en dressant explicitement la liste des qualités requises pour quiconque s'attaquerait à la traduction d'un chef-d'œuvre :

Tout d'abord, il doit avoir autant de talent - ou du moins la même forme de talent - que l'auteur qu'il a choisi. [...] Ensuite, il doit connaître parfaitement à la fois son pays et celui de son auteur, ainsi que tous les aspects du style et des méthodes de ce dernier, le contexte social des mots, leur vogue, ce qu'ils évoquaient jadis, ce qu'ils évoquent aujourd'hui. Enfin, outre son talent et son savoir, il doit posséder le don d'imitation, être capable de jouer le rôle de l'auteur, copiant fidèlement son comportement, son élocution, ses manières et sa forme d'esprit 11 .

En outre, le traducteur doit être aussi talentueux que l'auteur et il n'est pas fortuit que, parmi les traducteurs de qualité, Nabokov cite les écrivains de renom : Baudelaire, Poe, Joukovskij et Schiller.

À défaut de trouver un traducteur à leur hauteur, les écrivains plurilingues choisissent souvent de recréer euxmêmes leur œuvre dans une autre langue. C'est le cas de Samuel Beckett, de Nancy Huston et de bien d'autres. Nancy Huston l'a exprimé clairement quant à la réécriture/traduction de son roman Plainsong en français : «Je n'aurais fait confiance à personne pour le traduire. Quand la première version a été terminée, je l'ai réécrite en français ${ }^{12}$. » Samuel Beckett a également créé la plus grande partie de son œuvre en deux langues, en procédant à la traduction/réécriture en anglais de ses œuvres écrites en français et inversement. C'est aussi le cas d'Anne Weber qui écrit aujourd'hui en français et en allemand et qui s'autotraduit systématiquement ${ }^{13}$.

Nabokov, quant à lui, a trouvé sa propre stratégie qui s'est avérée infaillible, du moins pour les traductions du russe vers l'anglais. Afin de pouvoir intervenir à sa guise sur les traductions en anglais de ses œuvres russes, Nabokov préfère choisir des traducteurs jeunes et malléables qui acceptent docilement toutes ses interventions et corrections. Cela a par exemple été le cas de Michael Scammel, qui est un tout jeune étudiant au moment où Nabokov lui confie la

10. Pour certaines œuvres, notamment la traduction des nouvelles par Pertzoff, Maxim Shrayer estime la réécriture de Nabokov à la hauteur de $80 \%$. Voir M. Shrayer, « After Rapture and Recapture: Transformations in the Drafts of Nabokov's Stories ", dans The Russian Revew, ${ }^{\circ} 58$, octobre 1999, p. 557.

11. V. Nabokov, «L'art de la traduction », dans Littératures, Paris, Robert Laffont, 1985, p. 902.

12. D. Laurin, « Source sûre », entretien avec Nancy Huston dans Voir, 16-22 septembre 1993.

13. D. Weissmann, «"Trouver sa langue, trouver sa place": Anne Weber et l'idéal d'une littérature de l'entre-deux », dans R. Zschachlitz (dir.), Enjeux et perspectives d' un canon culturel européen. Au-delà des canons culturels et littéraires nationaux ?, Paris, L'Harmattan, 2012, p. 143-160. 
traduction en anglais de ses romans Le Don et La Défense Loujine :

Nabokov, à l'époque, venait de conclure son contrat avec Putnam pour publier plusieurs de ses romans russes en anglais. Au même moment, il fut privé de son traducteur attitré, en respectant le désir de son fils Dimitri de partir à Rome pour étudier l'opéra. En plus - et je n'ai pas compris ça à l'époque - Nabokov cherchait clairement quelqu'un de jeune et d'assez malléable (comme son fils, vraisemblablement) qui ne s'opposerait pas à une large réécriture à laquelle se livrait habituellement Nabokov lors de la révision de ses traductions. Comme cela arrive souvent, je fus, par hasard, la bonne personne qui se trouva au bon endroit au bon moment, ce qui fit économiser à Nabokov beaucoup de temps et lui évita bien des ennuis $[\ldots]^{14}$.

Au cours de cette période de treize ans, pour toutes ses traductions vers l'anglais, Nabokov procède de la même manière. Le traducteur lui fournit une traduction de base, une sorte de matrice textuelle littérale. Cette matrice, qui doit être la plus fidèle possible au texte original, sert à l'écrivain de point de départ pour un nouveau travail de création et de réécriture ${ }^{15}$.

La correspondance entre les traducteurs et l'auteur constitue une source d'information précieuse sur les processus créatifs sous-jacents à la traduction de ses œuvres. Les lettres de Nabokov à ses traducteurs sont souvent accompagnées de listes de plusieurs pages, voire de plusieurs dizaines de pages de commentaires où Nabokov note scrupuleusement toutes les corrections qu'il apporte au texte et dont le traducteur devra tenir compte lors d'une mise au net ultérieure.

L'implication de l'écrivain dans le processus de traduction a pu provoquer quelquefois des incidents « diplomatiques » avec certains de ses traducteurs. Nous y reviendrons. Mais ce que la correspondance présente de manière générale, les manuscrits permettent de l'observer dans le détail.

\section{Traduction de la nouvelle Ozero, oblako, bachnja} [Lac, nuage, tour] en anglais et en français

Prenons donc à présent un exemple concret, la nouvelle Озеро, облако, башня [Ozero, Oblako, Bachnja]. Cette nouvelle a été écrite par Nabokov en russe en 1937. Elle a été traduite en anglais sous le titre Cloud, Castle, Lake et en français sous trois titres différents :

Russe

Ozero, oblako, bachnja [Lac, nuage, tour].

Paru à Paris dans Sovremennye zapiski, 1937.

Anglais

Cloud, Castle, Lake.

Traduction par Peter Pertzoff en collaboration avec l'auteur, paru dans The Atlantic Monthly, $\mathrm{n}^{\circ} 167$, Boston, 1941 ; repris dans Nine Stories, 1948.

Français

a) Mirage

Traduit de l'anglais par un traducteur inconnu, Library of Congress, Manuscript Division, non publié.

b) Le Nuage, le lac, le château

Bibliothèque de Cornell University, traduit du russe par Vladimir Sikorski, paru dans $L^{\prime} A r c, \mathrm{n}^{\circ} 2,1964$.

c) Lac, nuage, château

Traduit de l'anglais par Maurice Couturier, NYPL, The Henry W. and Albert A. Berg Collection, paru dans V. Nabokov, Mademoiselle O, Paris, Julliard, 1982, réédité dans V. Nabokov, Nouvelles complètes, Paris, Gallimard, 2010.

Les traductions, à l'exception de la dernière, ont été supervisées par l'écrivain dans les moindres détails. Nous pourrons suivre la genèse de l'œuvre et de ses traductions grâce aux documents conservés au département

14. «Nabokov, meanwhile, had just concluded his contract with Putnam for several of his Russian novels to be published in English, and at the same time had lost his putative translator, his son Dmitri, owing to Dmitri's desire to travel to Rome to study opera. Beyond that - and I didn't understand this at the time - Nabokov was clearly looking for someone young and malleable enough (like his son, presumably) who wouldn't object to the extensive rewriting that Nabokov proposed to do in revising the translation. As so often happens, I chanced to be the right person in the right place at the right time, and it saved Nabokov a great deal of time and trouble to settle on me and press me into service» (M. Scammel, "Translation is a bastard form. An interview with Michael Scammel » (by Y. Leving), dans NOJ/HOЖ. Nabokov Online Journal, vol. I, 2007). 15. Cette exigence de fidélité au texte original défendue par V. Nabokov a fait couler beaucoup d'encre de la part des critiques littéraires et des spécialistes de traduction, et continue à les animer de nos jours. Voir, par exemple, J. Frances, «De Ars Translatio : Nabokov et la traduction », le 10 avril 2012 (http://jonathanfrances.wordpress.com/2011/04/10/de-arstranslatio-nabokov-et-la-traduction/) ou P. Assouline, « Nabokov, traducteur à géométrie variable », le 27 novembre 2009 (http://passouline.blog. lemonde.fr/2009/11/27/nabokov-traducteur-a-geometrie-variable/). 
des Manuscrits de la Bibliothèque du Congrès à Washington, de la New York Public Library et à la Bibliothèque de Cornell University (États-Unis) ${ }^{16}$. Ce corpus extraordinaire en trois langues nous offre la possibilité de comprendre ce qui motivait et influençait le travail créatif de Nabokov lors de la renaissance de ses œuvres en d'autres langues.

Bien fourni, le dossier génétique de la nouvelle s'organise de la manière suivante ${ }^{17}$ :

1) Ozero, oblako, bachnja [Lac, nuage, tour]

Première version manuscrite autographe, très corrigée par l'auteur (russe).

2) Ozero, oblako, bachnja [Lac, nuage, tour]

Seconde version imprimée dans la revue Sovremennye zapiski à Paris en 1937 (russe).

3) Cloud, castle, lake

Traduction dactylographiée par Peter Pertzoff avec des corrections manuscrites très importantes de l'auteur (anglais).

4) Cloud, castle, lake

Une mise au net dactylographiée avec quelques petites corrections par Véra Nabokov au crayon (anglais).

5) Cloud, castle, lake

Un poème, Song, traduit par Nabokov (anglais).

6a) Mirage

Traduit de l'anglais en français. Traduction dactylographiée avec quelques corrections minimes au crayon.

6b) Le Nuage, le lac, le château

Traduit du russe par Vladimir Sikorski ${ }^{18}$. Paru dans L'Arc, $\mathrm{n}^{\circ} 24,1964$.

6c) Lac, nuage, château (français)

Traduit de l'anglais par Maurice Couturier.

Photocopie d'une traduction dactylographiée, NYPL, The Henry W. and Albert A. Berg Collection.

Paru dans V. Nabokov, Mademoiselle O, Paris, Julliard, 1982 et rééditée dans V. Nabokov, Nouvelles complètes, Paris, Gallimard, 2010.

1) Ozero, oblako, bachnja [Lac, nuage, tour] première version manuscrite autographe, très corrigée par l'auteur (russe)

Le manuscrit de la nouvelle en langue russe montre comment Nabokov se livre à une campagne de correction et de réécriture importante (fig. 1). Ce document est constitué de neuf feuillets très fins, couverts d'une écriture relativement dense à l'encre noire très claire. Il contient au moins une campagne de correction et, d'après la comparaison avec d'autres dossiers génétiques de ses nouvelles, il semble qu'il s'agit du premier jet. On peut affirmer avec certitude qu'il n'y a pas eu de campagnes de réécriture sur d'autres supports, car ce document contient l'intégralité du texte qui sera publié quelques mois plus tard. La date du «25/26.6.1937 », qui est mentionnée sur le manuscrit (et lors de la publication), va dans le même sens en indiquant clairement que la nouvelle fut écrite en deux jours. Sur le premier feuillet, le titre est déjà présent, ainsi qu'un petit poème inscrit en biais en haut de la page, dans un espace qui restait disponible. Ce poème était destiné à être inséré au feuillet 4 , à la place des points de suspension laissés par l'écrivain en attente du poème à venir. Le dossier ne contient pas de brouillon de ce poème, qui a pourtant dû exister, car, malgré une correction, il semble s'agir d'une mise au net à partir d'un autre document. Cependant, nous ne pouvons pas l'affirmer avec certitude, car certains poèmes peuvent parfois être composés mentalement avant d'être jetés sur le papier sous leur forme quasi définitive.

2) Ozero, oblako, bachnja [Lac, nuage, tour] seconde version imprimée dans la revue Sovremennye zapiski à Paris en 1937 (russe)

Toutes les modifications introduites par Nabokov dans son brouillon sont reprises dans une version imprimée parue dans la revue Sovremennye zapiski à Paris en 1937 (fig. 2). Il faut noter que la nouvelle est publiée sous

16. Nous remercions D. Nabokov de son aimable autorisation de consulter les manuscrits de V. Nabokov. Nos remerciements vont également aux conservateurs des départements de manuscrits des Library of Congress, NYPL et Cornell University Library pour leur aide précieuse dans nos recherches.

17. Les documents 1 à 6 a sont conservés à la Library of Congress (Manuscript Division, Library of Congress, Washington, DC, Vladimir Nabokov Papers, Box 8) ; le document 6b est conservé à la Cornell University (Division of Rare and Manuscript Coll., Cornell University Library); le document $6 \mathrm{c}$ est conservé à The Henry W. and Albert A. Berg Collection, NYPL (New York).

18. Vladimir Sikorski est le fils d'Elena Sikorski (née Nabokov), sœur de Vladimir Nabokov. 


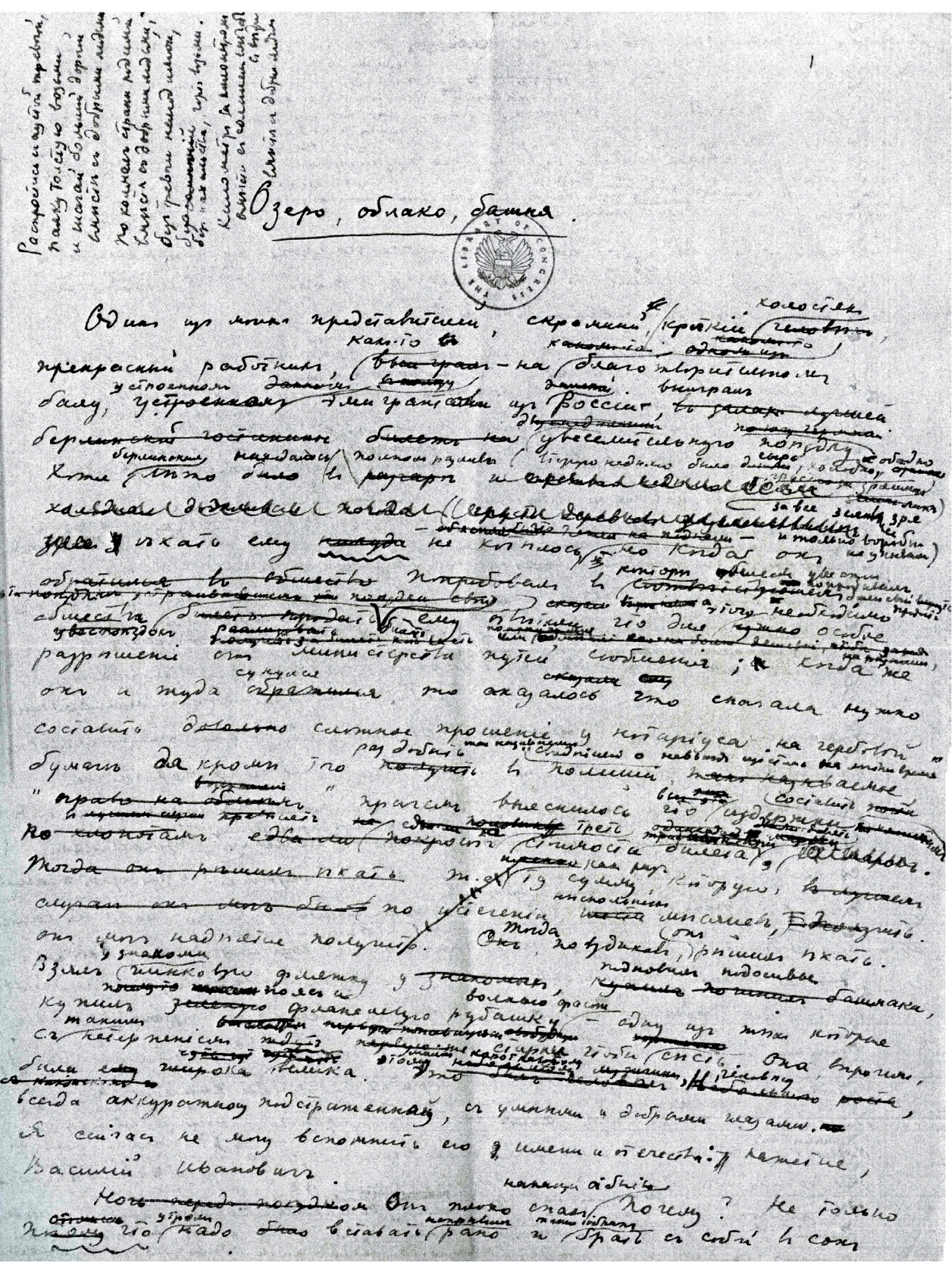

Fig. 1 : Ozero, oblako, bachnja, première version manuscrite autographe, corrigée par l'auteur (Library of Congress, Manuscript Division, Vladimir Nabokov Papers, Box 8, folder 22) 
le pseudonyme de Vladimir Sirine, avec lequel Nabokov signait toutes ses œuvres russes. En effet, on parle généralement de Vladimir Nabokov, écrivain russe et de Vladimir Nabokov, écrivain américain, or en réalité c'est le jeune écrivain Vladimir Sirine qui fut consacré meilleur écrivain russe du $\mathrm{XX}^{\mathrm{e}}$ siècle dans les années trente ${ }^{19}$.

\section{Oзеро, о6лако, башня}

Одюн из моих представителей, скромный, кроткій холостяк, прекрасный работник, как то на благотворительном балу, устроенном эмигрантами из Россіи, выиграл увеселительную поъздку. Хотя берлинское ль'то находилось в полном разливъ (вторую недъъю было сыро, холодно, обидно за все зеленъвшее зря, и только воробьи не унывали), ьхать ему никуда не хотйлось, но когда в. гонторь общества увеспоъздок он попробовал билет свой тродать ему отвоттил, что для этого необходимо (1) сcобое разрьшене ог мия когда же он и туда сунулся, то оказалось, что сначала нужно составить сложное протеніе у нотаріуса на тербовой бумагъ̆, да кромь того раздобыть в полищи так называемое «свиджттельство о невыъздъ нз торода на ль̆тнее время», при тем выяснилось, что издержки составят треть стоимости билета, т. е. как раз ту сумму, готорую, по истеченіи нйскольвих мйсяцев, он мог наrepror mor

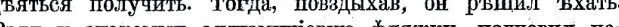
Взял у знакомых аллюоминіевую фляжкку, подновил подошвы, купил пояс и филанелевую рубапку вольнаго фасона, - одну нз тьх, которыя с таким нетерп安ніем ждут стирки, чтобы съ̌еть. Она, вщрочем, была велика этому милому, коротковатому человъку, всегда аккуратно подстриженному, с умными и добрыми глазами. Я сейчас не могу вспомнить его имя и отчество. Кажется, Василій Иванович.

Fig. 2 : Ozero, oblako, bachnja, seconde version imprimée dans la revue Sovremennye zapiski, Paris, 1937

3) Cloud, castle, lake - traduction dactylographiée par Peter Pertzoff avec des corrections manuscrites très importantes de l'auteur (anglais)

La nouvelle a été traduite du russe en anglais en 1941 par Peter Pertzoff. Mais, dès la première page, dès le titre même de la nouvelle traduit par Pertzoff comme A Lake, a cloud, a tower, on voit les interventions abondantes de Nabokov (fig. 3). Il est évident que le traducteur ne pouvait pas s'autoriser à prendre des libertés avec le titre et a choisi la traduction littérale du titre russe, en respectant ainsi la consigne de fidélité chère à Nabokov. Selon son habitude, l'écrivain modifie la version du traducteur en intervenant à deux niveaux : au niveau syntagmatique - il change l'ordre des mots, en mettant lake en dernière position - et au niveau paradigmatique - il remplace le substantif tower par castle, en obtenant ainsi une image sonore plus élégante ${ }^{20}$. Ainsi, le titre du traducteur A Lake, a cloud, a tower devient sous la plume de Nabokov Cloud, castle, lake. De toute évidence, Nabokov cherchait à recréer en anglais une chaîne phonologique forte semblable à celle du titre russe Ozero, oblako, bachnja $(o \rightarrow o b \rightarrow b)$. Le recours à deux phonèmes pivots $[k]$ et $[l]$ lui permet de construire une chaîne sonore tranchante, comme des coups de cravache, du titre anglais Cloud, castle, lake $(k l \rightarrow k \ldots l \rightarrow l . . k)^{21}$.

La nouvelle contient un autre exemple du travail de l'écrivain sur les allitérations, où il crée une très belle image sonore :

Traduction de Pertzoff

There was also a dark young man, by the name of Schram, with lusterless eyes and some vague a certain velvety odiousness in his appearance and manners, who constantly changed the conversation to one or another of the attractive aspects of excursion, and who gave the first signals for raptures ${ }^{22} \ldots$

\section{Traduction de Pertzoff corrigée par Nabokov}

There was also a dark young man, by the name of Schram, with lusterless eyes and a vague velvety vileness about his person and manners, who constantly switched the conversation to this or that attractive aspect of excursion, and who gave the first signal for rapturous appreciation..

19. Nous avons traité de la problématique du nom d'auteur pour V. Nabokov dans O. Anokhina, «Seuils des manuscrits chez Vladimir Nabokov », Genèse des seuils, Besançon, Presses universitaires de Franche-Comté, 2014 (sous presse).

20. Peut-être le terme castle était inspiré par le nom anglais d'une figure d'échec. Notons que la même figure, qui porte aujourd'hui en russe le nom de ladia, s'appelait avant la révolution de 1917 toura (-a étant en langue russe une marque du féminin).

21. Pour l'analyse détaillée de la transformation du titre, voir O. Anokhina, «Vladimir Nabokov : du style et des langues », La Licorne, $\mathrm{n}^{\circ}$ 98, « Dans l'atelier du style. Du manuscrit à l'œuvre publiée », dir. S. Bikialo et S. Pétillon, 2012, p. 211-220.

22. Ici et plus loin, les italiques indiquent les parties du texte qui ont été modifiées par l'écrivain. 


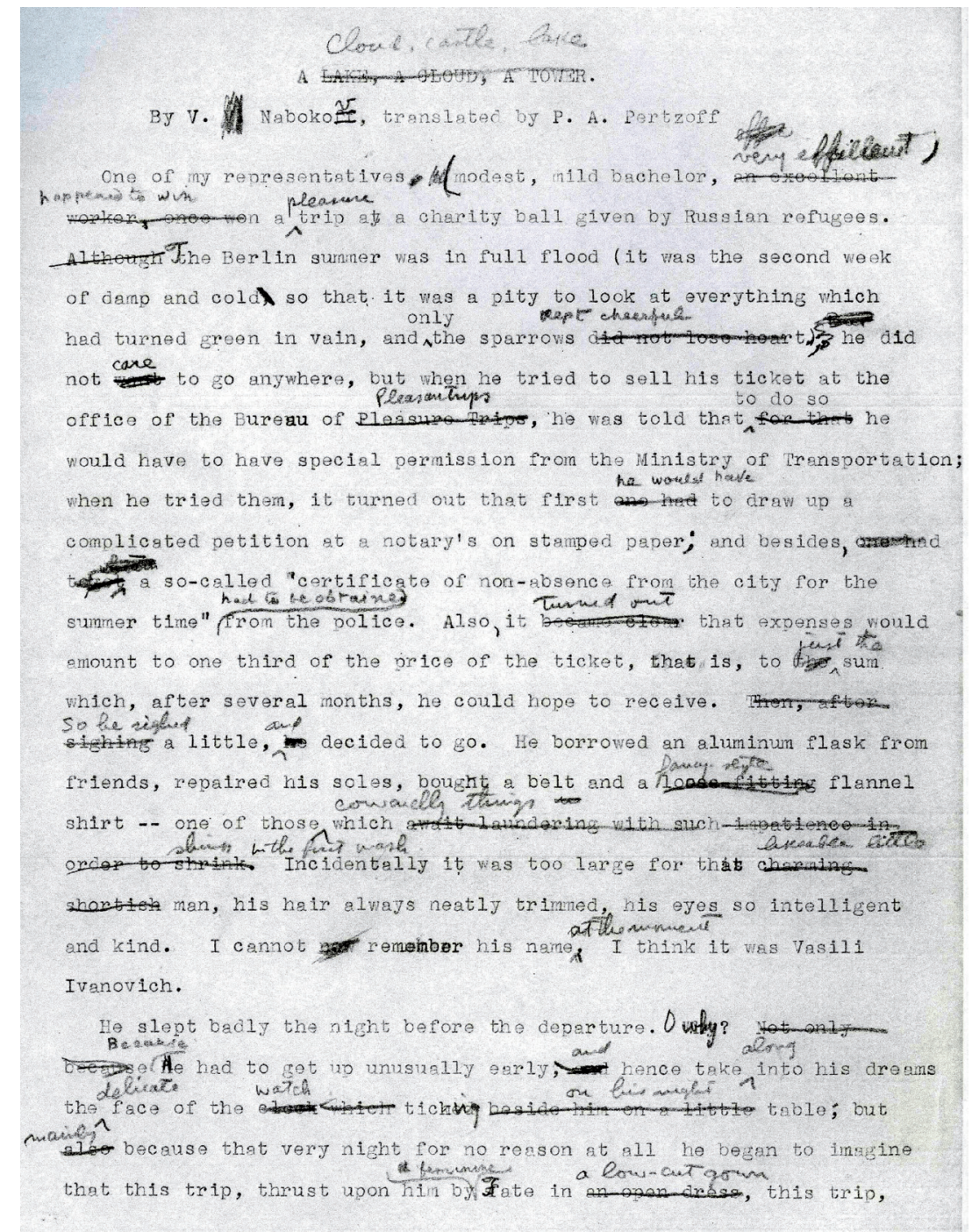

Fig. 3 : Cloud, castle, lake, traduction dactylographiée par Peter Pertzoff avec des corrections manuscrites de l'auteur (Library of Congress, Manuscript Division, Vladimir Nabokov Papers, Box 8, folder 25)

Ici, on voit comment une traduction faite par un autre (les termes vague et velvety sont déjà présents) inspire l'écrivain pour aller plus loin sur le plan créatif en fabriquant une allitération absente du texte russe original : vague velvety vileness. C'est également le cas de l'exemple suivant :

\section{Traduction de Pertzoff}

Trees appeared in groups and singly, turning calmly and smoothly, showing the new fashions.

\section{Traduction de Pertzoff corrigée par Nabokov}

Trees appeared in groups and singly, revolving coolly and blandly, displaying the latest fashions.

La traduction de cette phrase est particulièrement intéressante. Par la répétition du phonème $-l-$, Pertzoff crée ici une allitération absente du texte russe : singly, calmly, smoothly... Comme dans l'exemple précédent, Nabokov s'appuie sur cette trouvaille du traducteur pour la renforcer en apportant les modifications lexicales nécessaires : singly, revolving, coolly, blandly, displaying, latest.

Le même procédé se retrouve à la dernière page de la nouvelle :

\section{Traduction de Pertzoff}

Swept along a forset (sic) road in a wild fairy tale, squeezed, twisted, Vasili Ivanovitch could not even turn around, and only felt how the radiance behind his back receded, fractured by trees, and then it was no longer there, and all around the dark thickets aimlessly complained.

\section{Traduction de Pertzoff corrigée par Nabokov}

Swept along a forest road in a hideous fairy tale, squeezed, twisted, Vasili Ivanovitch could not even turn around, and only felt how the radiance behind his back receded, fractured by trees, and then it was no longer there, and all around dark firs fretted but could not interfere. 
Contrairement aux exemples précédents, où l'allitération surgit à partir des éléments fournis par le traducteur collaborateur, ici l'écrivain cherche à retrouver un effet stylistique qui s'est perdu lors d'une traduction, car le texte russe avait déjà une allitération à cet endroit : ponщyщцая чащцa :

Увцекаемый как в дикой сказке по месной дороге, зажатый, скрученный, Васимий Иванович не мог Ааже обернуться и только чувствовац, как сияние за спиной удацяется, Аробимое деревьями, и вот уже нет его, и кругом чернеет бездейственно ропщущая чаща.

La répétition de la consonne fricative $-f$ - sert à recréer en anglais le murmure de la forêt que la répétition du phonème chuintant $-w$ - rendait phonétiquement dans le texte russe.

L'importance des résonances phonologiques dans le travail de création a été soulignée par d'autres écrivains plurilingues :

[...] le rythme, la phonétique, c'est-à-dire la musique en général, pour moi sont primordiales. Donc souvent, j'ai été prête à sacrifier le sens précis des mots pour préserver un certain nombre de syllabes, ou pour préserver une allitération 23 .

Nous partageons l'opinion d'Emilio Sciarrino qui soutient que la prévalence du signifiant sur le signifié, de la forme sonore sur le contenu lexical est une caractéristique notoire propre au grand nombre d'écrivains plurilingues $^{24}$. Comme nous l'avons montré ailleurs 25 , ce travail sur la sonorité motivé par la recherche du «plaisir esthétique 26 » constitue chez Nabokov l'essence même de la réécriture de ses œuvres russes en anglais 27.

Le travail stylistique sur le titre provoque des répercussions sur le texte même de la nouvelle, Nabokov cherchant un moyen de modifier toute référence à la tour au profit du château :

\section{Traduction de Pertzoff}

On the other side, on a hill thickly covered with verdure (which was darkest where most poetic) there rose straight from dactyl to dactyl an ancient black tower.

\section{Traduction de Pertzoff corrigée par Nabokov}

On the other side, on a hill thickly covered with verdure (and the darker the verdure, the more poetic it is) towered arising from dactyl to dactyl an ancient black castle 28 .
En répondant à la nécessité de remplacer tour par château pour garder la cohérence avec les modifications apportées au titre, au-delà d'un simple changement lexical, Nabokov réussit un coup de maître en formant un adjectif, towered, en référence au castle. Il concilie ainsi l'idée de tour qui s'érige en hauteur et qui est présente dans la nouvelle en langue russe avec celle de château qui a émergé lors de son travail stylistique sur le titre de la nouvelle en anglais. Nous retrouvons ici un parfait exemple de « diamant », dont parle M. Shrayer en se référant au remarquable travail stylistique auquel se livre Nabokov lors de la traduction de ses œuvres en anglais : "Nabokov's editing of the translations of his three finest stories into English in the 1940s further testifies to the centrality of verbal perfectioning in his art. Nabokov's English diamonds are impeccably cut and polished ${ }^{29}$. »

En comparant le texte du traducteur, locuteur natif de langue anglaise, et celui révisé par l'écrivain, on constate que celui-ci est plus idiomatique. Cela peut paraître paradoxal, mais il est clair que le traducteur était paralysé par l'exigence de fidélité absolue posée par l'auteur. Par ailleurs, comme l'ont montré les exemples ci-dessus, Nabokov va beaucoup plus loin dans le sens de la créativité linguistique en anglais. Ainsi, il crée parfois de nouvelles images sonores qui sont absentes du texte russe (comme dans le cas de vague velvety vileness), parfois il continue le travail de créativité qui a déjà marqué le texte original en russe, en reconstituant une allitération non rendue par son collaborateur (firs fretted ... interfere). Ce travail peut également toucher la créativité

23. Nancy Huston, entretien avec C. Klein-Lataud, Société RadioCanada, 16 novembre 1993.

24. Emilio Sciarrino, «Effets du multilinguisme dans la poésie italienne contemporaine », dans « The Poetics of Multilingualism - La poétique du plurilinguisme », Colloque international, Eötvös Lorànd University, Budapest, 4-6 avril 2013 (à paraître).

25. O. Anokhina, «Vladimir Nabokov: du style et des langues », art. cit. 26. V. Nabokov, Postface de l'édition américaine de Lolita, 1958.

27. Nous avons pu développer cette analyse grâce au travail de Jane Grayson qui, durant de nombreuses années, fut la seule à avoir accès aux manuscrits de Nabokov et en a fait une analyse remarquable. Voir J. Grayson, Nabokov translated. A comparison of Nabokov's Russian and English Prose, Oxford, Oxford University Press, 1977.

28. V. Nabokov, Cloud, castle, lake, Library of Congress, Manuscripts Division, Box 8 , folder 25, p. 7

29. M. Shrayer, op. cit., p. 563. 
lexicale, comme c'est le cas de l'exemple suivant. Le texte russe contenait une très belle image du matin « fumant 30 »:

Утро поднялось пасмурное, но теплое, парное, с внутренним сомнцем..

Le traducteur l'a rendu littéralement, en respectant la structure syntagmatique de la phrase russe, alors que Nabokov s'aventure à créer un néologisme, steam-warm, adjectif qui englobe les notions de chaud et de fumant :

Traduction de Pertzoff

The morning turned out to be cloudy, but warm, steamy, with an inner sun..

\section{Traduction de Pertzoff corrigée par Nabokov}

The morning was dull, but steam-warm and close, with an inner sun...

Notons qu'aucune des trois traductions françaises dont il sera question plus loin ne pourra recréer l'originalité de cette image, ce «diamant» avec un éclat si particulier en langues russe et anglaise.

Outre ce travail stylistique d'orfèvre, l'écrivain méticuleux et perfectionniste traque les moindres imprécisions et oublis du traducteur. Un grand nombre de corrections relève de ce souci de l'écrivain de ne laisser s'évanouir aucun détail ni aucune image que contenait le texte russe. La première page de la traduction dactylographiée de Pertzoff (fig. 3) nous offre plusieurs exemples de ce travail sur le texte du traducteur qui manque par moments de rigueur. Ainsi, dans la première phrase de cette page, Nabokov tient à préciser qu'il ne s'agit pas d'un simple voyage, mais bien du pleasure trip, en rendant plus exactement l'expression увеселительная поездка [voyage d'agrément] du texte russe.

La traduction de Pertzoff rend également moins perceptible la difficulté que représentait l'obtention du certificat. Or, ce détail a son importance, car c'est précisément pour cette raison que le héros de la nouvelle consent finalement à effectuer le voyage. Nabokov rectifie la traduction en optant pour la structure passive de la phrase qui, en reliant l'expression d'obligation had to be obtained avec la mention d'une instance à solliciter, from the police, explicite une démarche longue, difficile et probablement vouée à l'échec :

\section{Traduction de Pertzoff}

[...] and besides one had to get a so-called "certificate of nonabsence from the city for the summer time" from the police.

Traduction de Pertzoff corrigée par Nabokov

$[\ldots]$ and besides, a so-called "certificate of non-absence from the city for the summer time" had to be obtained from the police.

La phrase traduite par Pertzoff, I cannot remember his name, I think it was Vasili Ivanovich (fig. 3), est grammaticalement correcte, mais son cadre temporel est moins précis que celui de la phrase russe : Я сейчас не могу вспомнить его имя и отчество. Кажется, Василий Иванович [Je n'arrive pas à me rappeler son nom en ce moment, je crois bien que c'était Vasili Ivanovitch]. Alors Nabokov ajoute un complément circonstanciel de temps, at the moment, renforçant ainsi la dimension narrative spatiotemporelle : I cannot remember his name at the moment, I think it was Vasili Ivanovich.

Dans la dernière phrase de cette page (fig. 3), Nabokov remplace le connecteur d'addition also par le connecteur explicatif mainly, plus approprié au sens exprimé dans le texte russe :

\section{Traduction de Pertzoff}

He slept badly the night before the departure. Why? Not only because he had to get unusually early, and hence take into his dreams the face of the clock which ticked beside him on a little table, but also because that very night for no reason at all he began to imagine that this trip, thrust upon him by fate in an open dress, this trip, which he had accepted so unwillingly, would bring him a wonderful, tremulous happiness.

Traduction de Pertzoff corrigée par Nabokov

He slept badly the night before the departure. $O$ why? Because he had to get unusually early, and hence take along into his dreams the delicate face of the watch ticking on his night table; but mainly because that very night for no reason at all he began to imagine that this trip, thrust upon him by a feminine Fate in an low-cut gown, this trip, which he had accepted so reluctantly, would bring him a wonderful, tremulous happiness.

30. L'adjectif fumant [парное] caractérise principalement le lait de vache qui vient d'être tiré et qui est donc encore chaud et fumant. 
Par ailleurs, l'écrivain remplace the face of the clock de Pertzoff par the delicate face of the watch. D'une part, il reconstitue une nuance affective de цичико часов [un petit visage de la montre/un petit visage du réveil]. D'autre part, en remplaçant le nom clock par watch, il apporte une précision que seul l'auteur pouvait effectuer, car le terme russe yacbı désigne indifféremment une montre, un réveil et une horloge. Quel terme choisir alors pour la traduction en anglais? Dans le contexte, le réveil semble plus approprié. De plus, clock étant un terme relativement générique (même s'il l'est dans une moindre mesure que le terme russe $y a c b \iota)$, le traducteur réussit à conserver l'opacité du terme russe. Néanmoins, malgré sa portée générique, le substantif clock désigne plus spécifiquement un réveil pour les anglophones. Par ailleurs, la caractéristique affective le petit visage semble quelque peu incongrue pour un réveil, mais convient parfaitement pour un objet de petite taille, une montre 31 . Nous reviendrons à ce terme plus loin, en relation avec les traductions de la nouvelle en français.

4) Cloud, castle, lake - mise au net dactylographiée avec quelques corrections au crayon par Véra Nabokov (anglais)

Un quatrième document est une mise au net de la traduction anglaise, qui inclut toutes les modifications apportées par l'auteur. Cette version contient quelques petites corrections manuscrites par Véra Nabokov au crayon (fig. 4). À la fin du tapuscrit, l'inscription «by V. Nabokov, translated by the author and P.A. Pertzoff » est corrigée au crayon de la manière suivante : «by V. Nabokov, translated from the Russian by P.A. Pertzoff and the author». Cette correction précise qu'il s'agit bien d'une traduction du russe - fait qui sera omis dans certaines publications - et met en avant le traducteur. Malgré ses exigences, sa «tyrannie 32 » envers ses traducteurs, Nabokov était extrêmement honnête avec eux dans les questions de reconnaissance officielle de leur travail et de rémunération, contrairement par exemple à Heinrich Heine ${ }^{33}$.

5) Cloud, castle, lake - un poème, Song, traduit par Nabokov (anglais)

Comme plusieurs textes en prose de Nabokov, la nouvelle intègre un petit poème que nous avons mentionné

plus haut. Fait peut-être moins connu par le grand public, Vladimir Nabokov a toujours eu une intense activité poétique. Les archives contiennent un nombre important de brouillons de ses poèmes. Autre fait notoire : Nabokov composait les vers principalement en russe. Toutefois, quand il s'agissait d'éléments poétiques au sein de traductions vers l'anglais ou le français, il proposait systématiquement sa propre traduction. C'est aussi le cas ici. Contrairement au traducteur qui a livré une traduction littérale, V. Nabokov opte pour un équivalent approximativement rimé :

Version du traducteur

Say farewell to empty fears,

Take a walking stick,

And march along the highway

With your fellow-men ${ }^{34}$.

Ce travail poétique confirme la tendance générale des traductions de Nabokov : le texte proposé par le traducteur, comme ici le poème, reste assez proche du texte original, alors que l'écrivain prend des libertés en composant un nouveau texte «poétique».

6a) Mirage - traduit de l'anglais, traduction dactylographiée avec corrections minimes au crayon (français)

6b) Le Nuage, le lac, le château - traduit du russe par Vladimir Sikorski, traduction dactylographiée avec des corrections manuscrites au crayon (français)

31. Pour d'autres exemples, voir M. Shrayer, «Cloud, Castle, Lake and the Problem of Entering the Otherword in Nabokov's Short Fiction », dans Nabokov Studies, 1994, vol. I, p. 131-153.

32. M. Couturier a évoqué - au sujet d'autre chose - la «tyrannie » de Nabokov. M. Couturier, Nabokov ou la Tyrannie de l'auteur, Paris, Éditions du Seuil, 1993.

33. À ce sujet, voir D. Weissmann, «From Staged to Disguised SelfTranslation. Heine and Celan in France », Arcadia, n ${ }^{\circ}$ 1, 2013, p. 436-445. 34. Le poème traduit par le traducteur est conservé à La Bibliothèque du Congrès (Box 8 , folder 25. Vladimir Nabokov Papers, Manuscript Division, Library of Congress, Washington, DC). 
Cloud, castle, lake.

One of ing representatives (modest, mild bachelor, very efficient) happened to win a pleasure trip at a charity ball given by Russian refugees. The Berlin summer was in full flood (1t was the second week of damp and cold, so that it was a pity to look at everything which had turned green in valn, and only the sparrows kept cheerful); he did not care to $\mathrm{gO}$ anywhere, but when he tried to sell his ticket at the office of the Bureau of Pleasantrips, he was told that to do so he would have to have special permission from the vinistry of Transportation; when he tried them, it turned out that first he would have to draw up a compl1cated petition at a notary's on stamped paper; and besides, a so-called "certificate of non-absence from the city for the sumuer time" had to be obtained froin the police. Also, it turned out that expenses would amount to one third of the price of the ticket, that is, to just the sum which, after several months, he could hope to receive. So he sighed a little, and decided to go. He borrowed an aluminum flask from friends, repalred his soles, bought a belt and a fancy-style flannel shirt - one of those cowardly things which shrink in the first wash. Incidentally, it was too large for that likeable little man, his hair always neatly trimed, his eyes so intelligent and kind. I cannot remember his nane at the moment. I think it was Vasili Ivanovich.

He slept badly the night before the departure. O why? Because he had to get up unusually early, and hence take along into his dreams the dellcate face of the watch tieking on his night table; but mainly because that very night for no reason at all he began to imagine that this trip, thrust upon him by a feminine Fate in a low-cut gown, this trip, which he had accepted so reluctantly, would bring him some wonder-

Fig. 4 : Cloud, castle, lake, mise au net dactylographiée avec quelques annotation de Véra Nabokov au crayon (Library of Congress, Manuscript Division, Vladimir Nabokov Papers, Box 8, folder 26)

6c) Lac, nuage, château - traduit de l'anglais par Maurice Couturier, photocopie d' une traduction dactylographiée 35 (français)

L'histoire de la traduction française semble un peu plus compliquée, car il existe trois traductions bien distinctes. Nous analyserons ici les deux premières (6a et 6b), que nous avons pu consulter. La première, qui se trouve aux archives de Washington, est intitulée Mirage. D'après la comparaison et l'analyse linguistique des versions anglaise et russe, nous pouvons affirmer qu'il s'agit d'une traduction à partir de l'anglais. C'est donc une traduction en français d'une traduction anglaise. Le lexique et même la syntaxe de cette traduction en français sont de toute évidence calqués sur la version anglaise :

\section{Cloud, Castle, Lake}

One of my representatives (modest, mild bachelor, very efficient) happened to win a pleasant trip at a charity ball given by Russian refugees. The Berlin summer was in full flood (it was a second week of damp and cold, so that it was a pity to look at everything which had turned green in vain, and only the sparrows kept cheerful)...

\section{Mirage}

L'un de mes représentants, - célibataire doux et plein de réserve, collaborateur précieux d'ailleurs -, vint à gagner un billet pour une excursion touristique, lors d'une fête de charité donnée par les réfugiés russes. Berlin était noyé sous les averses cet été-là, le froid et l'humidité persistaient depuis deux semaines déjà et c'était navrant de voir que toutes ses frondaisons avaient revêtu en vain leur plus verte parure seuls les moineaux demeuraient joyeux...

35. Nous n'étudierons pas cette traduction, effectuée après la mort de l'auteur à la demande de Véra Nabokov. 
Une traduction tout à fait autre fut publiée dans la revue L'Arc en 1964 sous le titre Le Nuage, le lac, le château. Le département des Manuscrits de Cornell University possède une copie dactylographiée de cette traduction avec quelques corrections manuscrites de l'auteur ${ }^{36}$. Fluide et élégante, la traduction de Vladimir Sikorski a été faite à partir de l'œuvre originale en russe :

Озеро, облако, башня/Ozero, oblako, bachnja

ОАин из моих представителей, скромный, кроткий холостяк, прекрасный работник, как то на благотворительном балу, устроенном эмигрантами из России, выиграл увеселительную поездку. Хотя берлинское мето находилось в полном разливе (вторую неделю было сыро, холодно, обидно за все зеленевшее зря, и только воробьи не унывами)..

\section{Le Nuage, le lac, le château}

En juillet 1935, un de mes représentants, travailleur infatigable, célibataire modeste et doux, a gagné à un bal de charité organisé par des émigrés russes un billet pour un voyage d'agrément. Bien que l'été berlinois fût en plein épanouissement (il faisait froid et humide depuis deux semaines déjà et c'était navrant de voir tout reverdir en vain ; il n'y avait que les moineaux pour ne pas perdre courage)...

La comparaison des deux traductions en français du premier paragraphe montre une relation étroite entre Mirage et la traduction anglaise Cloud, Castle, Lake. Ainsi, on y trouve en français vint à gagner, calqué sur l'expression anglaise happened to win, tout comme l'expression demeuraient joyeux est un calque transparent de kept cheerful. Le traducteur de Le Nuage, le lac, le château utilise quant à lui les termes neutres : gagner et ne pas perdre courage qui correspondent parfaitement aux expressions employées dans l'original russe.

Par ailleurs, on trouve dans Mirage le terme réfugiés (refugees dans le texte anglais), qui n'est pas particulièrement approprié en français. Ce mimétisme entre Mirage et la traduction anglaise se retrouve même dans la ponctuation : les parenthèses de la première phrase sont rendues par les tirets dans la traduction française ${ }^{37}$. La traduction Le Nuage, le lac, le château évoque quant à elle des émigrés russes, comme c'est déjà le cas dans l'original, terme plus approprié et largement utilisé pour caractériser le statut des Russes ayant émigré après la révolution de
1917. Cette dernière traduction ne recourt ni aux tirets ni aux parenthèses dans la première phrase pour la simple raison que ces signes de ponctuation sont absents de l'œuvre originale.

L'opacité du terme russe yacbl, que nous avons évoquée plus haut, et les traductions qui peuvent être proposées dans ce cas nous fournissent une preuve irréfutable du fait que la traduction en français Mirage prend sa source dans le texte anglais, alors que la traduction de Sikorski vient directement du russe. La traduction de Sikorski parle du «petit visage de réveil (личико часов) », alors que Mirage mentionne « la montre - joli visage aux traits délicats (the delicate face of the watch) - ». Il est donc évident que Sikorski n'a pas pris en compte dans son travail la traduction du russe vers l'anglais supervisée par Nabokov ${ }^{38}$, laquelle spécifie qu'il s'agit d'une montre, en traduisant à partir du russe directement (le terme réveil renvoyant au mot russe générique $y a c b \iota)$. En cela, Sikorski a respecté la tradition traductologique - que nous partageons - de traduire à partir d'une œuvre originale, mais malmené l'exigence de Nabokov que les traductions de ses œuvres doivent être faites à partir de la dernière traduction révisée par lui :

La traduction anglaise dûment polie à sa convenance, il demandait que les traductions suivantes en d'autres langues soient faites à partir de l'anglais plutôt que de l'original russe, non seulement parce qu'il y a beaucoup plus de traducteurs de l'anglais mais aussi parce qu'il considérait les versions anglaises, légèrement révisées, comme le texte définitif pour les lecteurs autres que russes 39 .

Même si les références archivistiques ne permettent pas de savoir quelle était la langue de départ pour la traduction de Sikorski, l'analyse linguistique nous amène à affirmer que, contrairement à Mirage, cette traduction est faite à partir d'une œuvre en langue russe.

36. Cornell University, Division of Rare and Manuscript Collections, Nabokov collection, box 10, folder 23.

37. Le changement de ponctuation lors d'une traduction est un sujet passionnant bien que peu exploré à ce jour.

38. Excepté le titre, comme nous le verrons par la suite.

39. B. Boyd, Vladimir Nabokov : les années américaines, Paris, Gallimard, 1999 , p. 534. 
Cependant, il est intéressant de noter que le titre de la nouvelle en français, Le Nuage, le lac, le château, fut quant à lui modifié en correspondance avec le nouveau titre anglais. On y voit la réintroduction des articles, ce qui change sa structure rythmique. Par ailleurs, l'ordre des noms est modifié par rapport à Ozero, oblako, bachnja [Lac, nuage, tour] en russe et Cloud, castle, lake [Nuage, château, lac] en anglais. Le point le plus marquant est que cette traduction a retenu l'équivalent de la traduction anglaise : château au détriment du nom employé dans le titre de l'œuvre originale : tour ${ }^{40}$. Nous retrouvons donc dans la traduction française les traces de la traduction vers l'anglais, même si la nouvelle en français puise son origine dans l'œuvre initiale écrite en russe. Il est intéressant de noter que, paradoxalement, la traduction de l'anglais vers le français de M. Couturier, que nous n'analysons pas ici, revient en partie au titre initial russe, en reprenant le même ordre des noms : Lac, nuage, château.

Ce détail laisse entrevoir le fait que les traductions d'une œuvre vers deux langues différentes peuvent parfois avoir une relation insoupçonnée. Cela nous fait penser au rôle peu connu qu'ont joué les traductions allemandes des pièces de théâtre de Beckett. Après avoir travaillé à la mise en scène de ses pièces en Allemagne et collaboré à leur traduction vers la langue allemande, qu'il maîtrisait très bien, Beckett cherchait systématiquement à intégrer les modifications apparues lors de ce travail aux versions anglaise et française de la même pièce 41 .

Le recours aux manuscrits de traductions apporte un éclairage nouveau quant au véritable processus créatif des écrivains plurilingues. Notons que Nabokov niait tout intérêt de recourir aux manuscrits, alors même qu'il a soigneusement constitué, entretenu et catalogué des archives conséquentes contenant les manuscrits de ses œuvres en différentes langues, les manuscrits de traductions, ainsi que la correspondance :

Rough drafts, false scents, half explored trails, dead ends of inspiration, are of little intrinsic importance. An artist should ruthlessly destroy his manuscripts after publication, lest they mislead academic mediocrities into thinking that it is possible to unravel the mysteries of genius by studying canceled readings. In art, purpose and plan are nothing; only the results count 42 .

Cependant, dans le cas de Nabokov, l'existence des brouillons de traductions, comme nous venons de le voir pour la nouvelle Ozero, oblako, bachnja, offre aux chercheurs le privilège de comprendre les relations parfois complexes entre une œuvre originale et ses traductions en d'autres langues.

\section{Stratégies d'intervention de Vladimir Nabokov sur les traductions de ses œuvres}

L'observation des manuscrits de traducteurs corrigés par l'écrivain permet de distinguer chez Nabokov différentes stratégies d'intervention. Quand il s'agit d'une traduction vers l'anglais, ses interventions sont très importantes et portent autant sur le lexique que sur la syntaxe. Il n'hésite pas à réécrire des phrases et des paragraphes entiers. Le travail sur la sonorité que nous avons évoqué plus haut occupe également une place importante.

Quand il s'agit de traductions vers le français, que ça soit du russe ou de l'anglais, les modifications de l'auteur portent principalement sur le lexique. Ses corrections peuvent alors être accompagnées de commentaires métadiscursifs qui constituent une sorte de dialogue différé avec le traducteur. Ce type de corrections abonde dans le dossier génétique de la traduction française de Pale Fire [Feu pâle].

Le roman Feu pâle fut traduit par Raymond Girard et Maurice-Edgar Coindreau et publié à Paris par Gallimard en 1965. La dactylographie de la traduction avec de très nombreuses corrections et annotations de Nabokov se trouve au département des Manuscrits de NYPL.

Même si, pour cette traduction de Feu pâle, les révisions de Nabokov touchent uniquement le lexique, ses interventions ont provoqué une véritable guerre froide déployée dans les lettres entre l'auteur et les traducteurs ${ }^{43}$. Le traducteur exigeant a refusé catégoriquement d'apporter au

40. Cependant, si le texte français Mirage parle bien du château, le texte de Sikorski laisse la référence à une tour noire, preuve supplémentaire de sa parenté avec le texte russe.

41. D. Weissmann, "Writing from the midzone" : réflexions sur la territorialité de l'écrivain plurilingue auto-traducteur », dans C. Oster et R. Jenn (dir.), Translating Territories/Territoires de la traduction - Traduction du territoire, Arras, Artois Presses université, 2014 (sous presse).

42. V. Nabokov, «Translator's Introduction », dans Aleksandr Pushkin, Eugene Onegin: A Novel in Verse, Princeton, Princeton University Press, 1975 , p. 15.

43. B. Boyd analyse cet incident et donne l'exemple de quelques termes qui ont posé problème lors de cette traduction. Voir B. Boyd, op. cit., p. $528-529$ 
texte de sa traduction une quelconque modification suggérée par l'auteur :

p. 2 : [...] Soyez sûr que nous commettons nous-mêmes assez d'erreurs sans que vous nous infligiez à en ajouter.

p. 3 : [...] Passons aux barbarismes. P. 78 vous aimeriez que le mot dim soit traduit par brumailleux. Ce mot malheureusement ne figure pas dans mes dictionnaires. Or, nous avons suffisamment de termes pour désigner un pays 'dim' (brumeux, enfumé, etc.) sans qu'il soit nécessaire d'en forger un nouveau.

Enfin, ainsi que je vous le disais tout au début de cette lettre, nous ne pouvons accepter des phrases ou des mots qui prêteraient au rire, ou qui seraient d'une telle gaucherie que nos lecteurs seraient en droit de protester.

p. $3:[\ldots]$ Ce n'est pas à l'âge de soixante et onze ans que je vais me mettre à entériner des obscénités.

Je n'ai pas publié trente-trois traductions sans avoir appris qu'un traducteur a des devoirs impérieux envers l'auteur de l'œuvre qu'il traduit. Il doit le consulter pour éviter les contresens, les erreurs d'interprétation, pour résoudre des problèmes insolubles et qui obligent à des pis-allers de fortune. C'est le cas pour les jeux de mots, calembours qui abondent dans Pale fire. En ce cas, les solutions que vous proposerez seront les bienvenues. Mais les devoirs de traducteurs s'arrêtent là où commencent les devoirs de l'auteur, devoirs qui peuvent se résumer en un seul : laisser le traducteur maître de sa syntaxe et de son vocabulaire. Le traducteur écrit, en général, dans sa langue maternelle qu'il connaît toujours mieux que ne la connaît l'auteur du livre qu'il traduit. [...] Il ne peut donc pas accepter d'abaisser le niveau d'une seule de ses œuvres en accueillant, les yeux fermés, des suggestions erronées. Veuillez donc vous limiter à corriger les fautes de sens que j'ai certainement commises. [...] Je n'apporterai donc aucune modification à mon texte, à moins qu'il ne s'agisse de contresens flagrant.

(Lettre de Maurice-Edgar Coindreau, cotraducteur de Feu pâle en français, à Nabokov, 6 janvier 1964, département des Manuscrits, NYPL)

On peut imaginer l'indignation de l'écrivain habitué à réécrire les traductions de ses œuvres :

Vous me dites, Monsieur, que vous avez publié 33 traductions. J'ai eu, moi, plus de soixante traducteurs. Avec beaucoup d'entre eux j'ai collaboré étroitement. Donc, comme vous, j'ai acquis une certaine habitude.

En lisant votre lettre dont le ton m'a paru unfriendly and quite uncalled for $[\ldots]$

p. 5 : Quant au rire du lecteur, on ne le provoquera pas si seulement mes traducteurs veulent bien s'entendre avec moi.
[...] Comme jusqu'ici je ne me suis permis aucune atteinte à votre style (ou à celui de M. Girard), la violence de vos parades dans le vide ne me semble pas justifiée. Vous me parlez de vos droits de traducteur. Ils sont indiscutables. Néanmoins il existe des borderline cases où nos droits se touchent.

(Réponse de Nabokov à Maurice-Edgar Coindreau, cotraducteur de Feu pâle en français, le 14 janvier 1964, département des Manuscrits, NYPL)

Nabokov a également vivement protesté auprès de l'éditeur, en réclamant le droit de regard sur ses œuvres traduites :

Je ne veux plus entendre Coindreau dire que je suis coupable de barbarismes. Alors qu'il est coupable de ne pas être précis. La seule chose importante est que mon français est suffisant pour voir plus précisément que lui que son français ne correspond pas à mon anglais. En tout cas, je dois voir les épreuves et ce qui est le plus important, c'est que je dois avoir le dernier mot pour décider de telle ou telle traduction, même si ce dernier mot doit être un compromis courtois 44 .

(Lettre de Nabokov à Gallimard (Michel Mohrt), le 19 janvier 1966, département des Manuscrits, NYPL)

\section{L'autotraduction comme perpétuation du processus créatif}

En conclusion, nous voudrions attirer l'attention sur une particularité des traductions de Nabokov.

Après avoir assis sa gloire d'écrivain américain, Nabokov s'est employé à introduire dans ses traductions un nombre croissant de références linguistiques et culturelles russes, françaises et allemandes. En introduisant ces références, de même qu'en créant des personnages d'émigrés qui, au cours d'une discussion, naviguent entre l'anglais, le russe et le français (tout comme Nabokov le faisait luimême dans sa correspondance), l'écrivain semble vouloir souligner le caractère cosmopolite de son œuvre et de son identité personnelle.

Le multilinguisme inhérent à ses œuvres constitue un véritable défi pour leur traduction. Ainsi, comment traduire en français le roman écrit en anglais Ada or Ardor mais qui utilise également de façon très significative le

44. C'est nous qui soulignons. 
français et le russe 45 ? Cette difficulté fut exprimée par Jacques Tournier, chargé de la traduction française du roman :

À ce propos, et d'une manière plus générale, il faut savoir que l'un des charmes de l'écriture de ce roman tient à la virtuosité avec laquelle l'auteur joue, en même temps, de trois langues différentes : l'anglais, le français et le russe, et les fait alterner, comme un peintre jouant des trois couleurs. Traduire ce livre en français, c'est perdre un des trois langages, ne plus se servir que de deux couleurs. C'est renoncer à un certain jeu de facettes, d'éclats, de scintillement et de surprises dans l'écriture ellemême, quelles que soient les équivalences proposées 46 .

Quelle était la réponse de Nabokov à cette requête passionnée ? Il a marqué en marge au crayon rouge un impitoyable verdict : «Renoncer ! La fidélité avant tout ».

L'écrivain a souligné plus d'une fois l'importance de la fidélité à ses œuvres originales, comme dans cette préface à la traduction anglaise de la nouvelle Invitation au supplice/ Invitation to a Beheading [Приглашение на казнь] :

En 1935, mon texte russe avait donné corps à une certaine vision et ce dans les termes précis qui lui convenaient. Les seules corrections dont pourrait profiter la version anglaise ne relevaient que de la simple routine, pour la recherche de cette clarté qui, en anglais, paraît exiger des traits électriques moins étudiés qu'en russe. Mon fils s'est révélé un traducteur d'une merveilleuse fidélité et il fut décidé entre nous que la fidélité à l'auteur choisi doit primer, si bizarre que puisse être le résultat. Vive le pédant !

Cette fidélité, exigée de ses traducteurs par l'écrivain, constituait une condition nécessaire lui ouvrant le champ de la créativité. Les traductions des œuvres de Nabokov, et surtout les documents de travail, offrent la possibilité de voir qu'un nouveau processus créatif s'enclenchait pour l'écrivain à partir d'une matrice traduite par d'autres, car « un texte autotraduit [...] contient souvent des façons de "rendre" l'original qu'aucun traducteur - hormis l'auteur lui-même - ne se serait raisonnablement jamais aventuré à envisager ${ }^{47} \gg$. De ce fait, le travail de traduction de Nabokov entre dans la catégorie de l'« autotraduction recréatrice » définie par M. Oustinoff 48 .

Les généticiens ont maintes fois souligné que la question de l'achèvement ${ }^{49}$ est épineuse et que le processus créatif, du moins chez certains écrivains, ne s'arrête jamais, à moins que cet arrêt ne soit imposé par des impératifs éditoriaux ou autres. Un écrivain bilingue réussit à déjouer cette contrainte car si son œuvre est éditée, donc « figée », dans une langue, il peut la recréer, la modifier à loisir dans une autre langue par le biais de l' « autotraduction ».

Toute traduction, qu'elle soit allographe ou autographe, est une création qui engage des opérations et des processus créatifs ; une autotraduction, faite par l'auteur lui-même (Beckett, Weber), ou une traduction révisée et retravaillée par l'écrivain (Nabokov) qui s'en rapproche constituent incontestablement un type de création particulier. À la différence d'un écrivain unilingue qui, à un moment ou à un autre, se voit obligé d'interrompre son travail d'écriture pour entamer la phase éditoriale 50 , l'autotraduction recréatrice l'inscrit dans la continuité du processus créatif. L'utilisation de langues différentes comme supports d'écriture laisse aux écrivains plurilingues une plus grande marge de liberté créative : «À partir du moment où ils se traduisent eux-mêmes, les traductions ainsi réalisées font donc, par le fait même, texte ${ }^{51} »$. Ainsi, la « clôture » du texte, toujours artificielle et douloureuse pour un écrivain, est levée et le processus créatif est de nouveau enclenché : « le texte traduit peut dépasser l'original par ses qualités artistiques et esthétiques, car son auteur le retravaille en apportant une réflexion supplémentaire au moment de la traduction 52 ». En se livrant à l'autotraduction, les écrivains plurilingues s'octroient le droit de retarder le moment où le cordon ombilical avec leur œuvre sera coupé et où elle pourra acquérir une existence autonome, en établissant ainsi un « continuum créatif 53 ».

45. Sur les difficultés que présente la traduction d'une œuvre plurilingue, voir O. Anokhina, «Comment traduire les œuvres plurilingues : le cas Nabokov », dans Actes du colloque international « Traduction, plurilinguisme et langues en contact : traduire la diversité », Université de Toulouse-Le Mirail, 18-20 octobre 2012 (à paraître).

46. Commentaires de Jacques Tournier pour sa traduction de Ada or Ardor (département des Manuscrits, NYPL).

47. M. Oustinoff, Bilinguisme d'écriture et auto-traduction, Julien Green, Samuel Beckett, Vladimir Nabokov, Paris, L'Harmattan, 2001, p. 7.

48. Ibid.

49. L. Hay (dir.), Le Manuscrit inachevé. Écriture, création, communication, Paris, Éditions du CNRS, 1986.

50. P.-M. de Biasi, La Génétique des textes, Paris, Armand Colin, p. 44-49. 51. M. Oustinoff, op. cit., p. 21. C'est l'auteur qui souligne.

52. S. Garziano-Vassilieva, « La poétique autobiographique de Vladimir Nabokov dans le contexte de la culture russe et occidentale », Thèse de doctorat, Lyon, Université Jean-Moulin-Lyon III, p. 124

53. M. Shrayer, op. cit., p. 563. 
OLga ANOKHina est linguiste, chercheur à l'ITEM (CNRS-ENS) depuis 2002. Elle consacre ses recherches à la genèse des œuvres des écrivains multilingues. Olga Anokhina s'intéresse également aux aspects cognitifs de la production écrite, du multilinguisme et de la création. Elle a initié et dirigé en 2005-2007 un programme franco-russe « Multilinguisme et genèse des textes » (CNRS/Académie des sciences de Russie). Les résultats de ces recherches ont été publiés en 2012 dans un volume collectif, Multilinguisme et créativité littéraire chez Academia-Bruylant. Olga Anokhina dirige à l'ITEM l'équipe « Multilinguisme, traduction, création ».

olga.anokhina@freesbee.fr

\section{Résumés \\ Traduction et réécriture chez Vladimir Nabokov : genèse d'une œuvre en trois langues}

Nabokov écrivait, traduisait et s'autotraduisait en trois langues russe, anglais, français. Au fil des années, l'écrivain a élaboré sa propre stratégie pour la traduction de ses œuvres : il confiait la traduction de ses nombreuses fictions écrites en russe aux traducteurs professionnels de langues anglaise ou française et les supervisait ensuite personnellement en y apportant les modifications qu'il jugeait nécessaires. En se livrant à un travail important de révision et de réécriture, Nabokov prolongeait ainsi le processus créatif. La nouvelle Ozero, oblako, bachnja, écrite par Nabokov en russe, sert ici à illustrer l'approche. Cette œuvre fut traduite en anglais sous le titre Cloud, Castle, Lake par P. Pertzoff, puis revue par Nabokov dans les moindres détails. De ce texte, existent aussi trois traductions en français. Ce dossier génétique permet de comprendre ce qui motivai et influençait le travail créatif de Nabokov lors de la renaissance de son œuvre dans une autre langue.

Nabokov wrote, translated and self-translated in three languages: Russian, English and French. Over the years he elaborated his own strategy for the translation of his works: he would assign the translation of his numerous fiction works written in Russian to professional English or French translators and he would then personally supervise them and amend them. With this important revision and rewriting work Nabokov would thus prolong the creative process. To illustrate this approach we will use his short story Ozero, oblako, bachnja, written in Russian. This work was translated in English by P. Pertzoff under the title Cloud, Castle, Lake, and then revised in great detail by Nabokov. There also exists three French translations of this text. This genetic dossier allows us to understand what motivated and influenced Nabokov's creative work during the rebirth of his writings in another language.

Nabokov schrieb, übersetzte und übersetzte sich selbst in drei Sprachen - Russisch, Englisch und Französisch. Im Verlauf der Jahre hat der Schriftsteller seine eigene Strategie für die Übersetzung seiner Werke ausgearbeitet: Er vertraute die Übersetzung seiner zahlreichen auf russisch verfassten Fiktionen professionellen Übersetzern für Englisch oder Französisch an, beaufsichtigte diese dann persönlich und brachte die Modifikationen an, die er für nötig hielt. Indem er sich dieser wichtigen Arbeit der Revision und Überarbeitung aussetzte, verlängerte Nabokov den kreativen Prozess. Die 1937 auf Russisch geschriebene Novelle Ozero, oblako, bachnja verdeutlicht diesen Vorgang: Das Werk wurde von P. Pertzoff unter dem Titel Cloud, Castle, Lake ins Englische übersetzt und die Übersetzung wurde vom Autor selbst bis ins kleinste Detail überprüft. Von diesem Text gibt es auch drei Übersetzungen ins Französische. Dieses genetische Dossier ermöglicht es zu verstehen, was Nabokovs kreative Arbeit während dieser Wiedergeburt seines Werkes in einer anderen Sprache motiviert und beeinflusst hat.
Nabokov escribía, traducía y se autotraducía en tres lenguas: en ruso, en inglés y en francés. A lo largo de los años, el escritor elaboró su propia estrategia para la traducción de sus obras: confiaba la traducción de sus numerosas ficciones escritas en ruso a traductores profesionales de lenguas inglesa y francesa, las corregía luego aportando las modificaciones que él pensaba necesarias. Al realizar así este importante trabajo de revisión y reescritura, Nabokov prolongaba así el proceso creativo. La novela Ozero, oblako, bachnja [Lago, nube, torre], escrita en ruso por Nabokov en 1937, ilustra este proceso: la obra fue traducida al inglés, bajo el título Cloud, Castle, Lake por P. Pertzoff y la traducción fue revisada por el escritor mismo con mucho cuidado. Existen también tres traducciones en francés. Este dossier genético plurilingüe nos permitirá comprender lo que motivaba e influía el trabajo creativo de Nabokov en este renacer de la obra en otro idioma.

Nabokov escrevia, traduzia e autotraduzia-se em três línguas russo, inglês, francês. Ao longo dos anos, o escritor desenvolveu uma estratégia própria para a tradução de suas obras: confiava a tradução das ficções escritas em russo a tradutores profissionais de inglês ou francês, que depois supervisionava pessoalmente introduzindo emendas. Com este trabalho extenso de revisão e reescrita, Nabokov prolongava assim o processo criativo. A novela Ozero, oblako, bachnja, escrita em russo, serve aqui para ilustrar o seu método. A obra foi traduzida para inglês sob o título de Cloud, Castle, Lake (Nuvem, Castelo, Lago) por P. Pertzoff, e então minuciosamente revista por Nabokov. Deste texto existem também três traduções em francês. Com um dossier genético deste tipo é possível compreender o que motivava e influenciava o trabalho criativo de Nabokov aquando do renascimento da sua obra em outro idioma.

Nabokov ha scritto, tradotto (e si è auto-tradotto) in tre lingue russo, inglese, francese. Nel corso degli anni, lo scrittore ha sviluppato una propria strategia per la traduzione delle sue opere. I suoi testi scritti in russo, ad esempio, affidati dapprima a traduttori professionisti di lingua inglese o francese, venivano quindi licenziati dopo un'opera di revisione e riscrittura che di fatto finiva per prolungarne il processo redazionale. La vicenda della novella Ozero, oblako, bachnja, composta da Nabokov in russo, diviene in questo saggio lo specimen significativo per intendere proprio quella dinamica creativo-evolutiva. La traduzione inglese della novella, realizzata, con il titolo Cloud, Castle, Lake, da P. Pertzoff, venne poi meticolosamente perfezionata da Nabokov. Di tale testo si conservano anche tre traduzioni in francese. Un dossier genetico, questo, che ci aiuta ad entrare nel sistema dell'autore, cioè nelle ragioni e nelle tecniche del suo lavorìo creativo in un'altra lingua. 